\title{
Expansion of lower-frequency locally resonant band gaps using a double-sided stubbed composite phononic crystals plate with
}

\section{composite stubs}

Suobin $\mathrm{Li}^{1}$, Tianning Chen ${ }^{1}$, Xiaopeng Wang ${ }^{1}$, Yinggang $\mathrm{Li}^{2}$, Weihua Chen ${ }^{1}$

1School of Mechanical Engineering and State Key Laboratory for Strength and Vibration of Mechanical Structures, Xi'an Jiaotong University, Xi'an, Shaanxi 710049, China.

2Key Laboratory of High Performance Ship Technology of Ministry of Education, Wuhan University of Technology, Wuhan, 430070, China

E-mail: xpwang@mail.xjtu.edu.cn

Abstract: We studied the expansion of locally resonant complete band gaps in two-dimensional phononic crystals (PCs) using a double-sided stubbed composite PC plate with composite stubs. Results show that the introduction of the proposed structure gives rise to a significant expansion of the relative bandwidth by a factor of 1.5 and decreases the opening location of the first complete band gap by a factor of 3 compared to the classic double-sided stubbed PC plate with composite stubs. Furthermore, more band gaps appear in the lower-frequency range (0.006). These phenomena can be attributed to the strong coupling between the "analogous rigid mode" of the stub and the anti-symmetric Lamb modes of the plate. The "analogous rigid mode" of the stub is produced by strengthening the localized resonance effect of the composite plates through the double-sided stubs, and is further strengthened through the introduction of composite stubs. The "analogous rigid mode" of the stubs expands the out-of-plane band gap, which overlaps with in-plane band gap in the lower-frequency range. As a result, the complete band gap is expanded and more complete band gaps appear.

\section{Introduction}

In the last two decades, acoustic wave propagation in periodic composite materials— known as phononic crystals (PCs) - has attracted much attention due to their unique physical properties. For example, phononic band gaps exist where acoustic wave propagation is forbidden ${ }^{[1-7]}$. There are two mechanisms that lead to the formation of band gaps, known as Bragg scattering and local resonance. Band gaps are generated by Bragg scattering and their wavelengths are the same order as the periodic structure. The widths and positions of Bragg band gaps depend heavily on the difference between the elastic parameters of the scattering and the host material, as well as the geometric parameters and the shape of inclusions ${ }^{[8-11]}$. A large lattice constant is needed to obtain low frequency band gaps, which hinders the application of PCs in the audible frequency range. On the other hand, the associated wavelength of band gaps generated by local resonance-which was first proposed by Liu at el. ${ }^{[12]}$ - is two orders of magnitude smaller than Bragg band gaps. A resonant band gap is related to the resonance frequency associated with scattering units, and depends less on the period and symmetry of the structure. This breaks the Bragg band gap limit and allows for low-frequency band gaps. As a result of this work, there has been much research on formation mechanisms of locally resonant band gaps ${ }^{[13-29]}$. However, little attention has focused on the expansion of these band gaps into the low-frequency range. Wu et al. ${ }^{[30]}$ demonstrated that 
locally resonant band gaps can appear in two-dimensional PC plates composed of a square array of stubs on one side of a homogeneous plate. They demonstrated that low-frequency band gaps can be obtained when stub height was roughly three times the plate thickness. Based on their work, many studies have concentrated on the expansion of locally resonant band gaps using PCs based on a similar geometry. Bilalet al. ${ }^{[31]}$ studied the band structure of a PCs formed by a periodic array of holes on one side of a stubbed plate. They demonstrated that a significant expansion of band gaps were obtained due to the trampoline effect. Li et al. ${ }^{[32]}$ combined those ideas and proposed an original acoustic metamaterial plate composed of a square array of stubs on one side of a $2 \mathrm{D}$ locally resonant PC plate. The results indicated that the combination of these structures lead to a significant expansion of the relative bandwidth in comparison to the classic one-sided stubbed plate (by a factor of 3). Nevertheless, all these studies focused on structures composed of a square array of stubs on one side of a finite thickness plate(either a homogeneous plate or a two-dimensional binary localized resonant PC plate). Assouar et al. ${ }^{[33]}$ studied locally resonant band gaps in a double-sided stubbed PC plate composed of a square array of composite stubs on two sides of a homogenous plate. In that study, the bandwidth was increased by a factor 2 compared to the classic one sided stubbed plates. Based on their study, Zhao et al. ${ }^{[34]}$ investigated the flexural vibration band gaps of this PC plate, and found that the bandwidth of the flexural vibration band gaps was obtained.

In this paper, we present an original structure composed of a square array of composite stubs on both sides of a composite plate. In this manner, the width and location limitations of the local resonance band gap can be overcome. A theoretical analysis and a numerical computation are used to investigate the physical behavior of the proposed PC plate, and to compare the behavior to the classic double-sided stubbed PC plates with composite stubs. We demonstrate that more band gaps appear, and that the relative bandwidth is expanded by a factor of 1.5. Furthermore, the opening location of the first band gap is reduced by a factor of 3 .

\section{Numerical results and discussion}

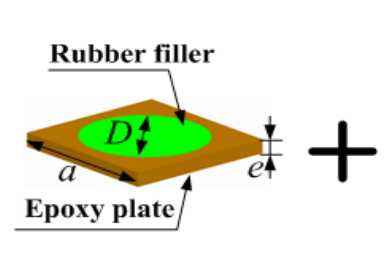

(a)

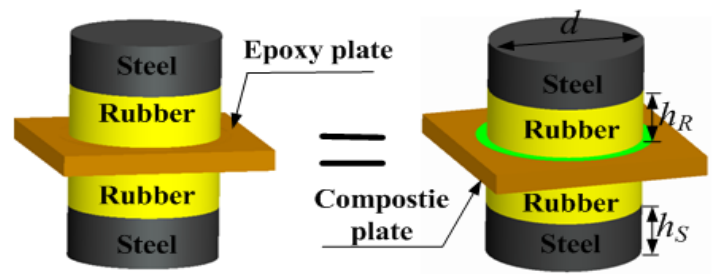

(b)

(c)

Fig. 1. Illustration of a unit cell of the proposed structure: (a) the composite plate; (b) the classic structure, a double-sided stubbed PC plate with composite stubs; and (c) the proposed structure, a double-sided stubbed composite PC plate with composite stubs.

The unit cell of the proposed structure is displayed in Fig. 1(c); it is formed by depositing composite stubs on both sides of a composite plate. The composite plate is fabricated by filling the drilled holes of a finite thickness epoxy plate with rubber filler, as shown in Fig. 1(a). The classic

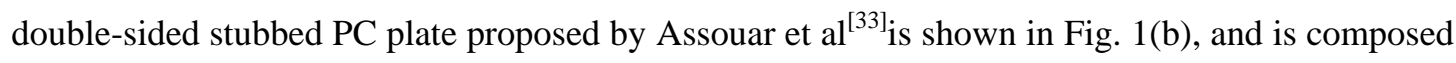
of composite stubs deposited on a homogeneous plate. $D, e$, and $a$ are the rubber filler diameter, the epoxy plate thickness, and the lattice constant, respectively. The height and diameter of the stub are denoted by $h$ and $d$, respectively. Note that $h=h_{R}+h_{S}$, where $h_{R}$ is the height of rubber stub, and $h_{S}$ is the height of the steel stub. 
TABLE 1. Material parameters in calculations.

\begin{tabular}{cccc}
\hline \hline Material & $\begin{array}{c}\text { Mass } \\
\text { Density }\left(\mathrm{kg} / \mathrm{m}^{3}\right)\end{array}$ & $\begin{array}{c}\text { Young's } \\
\text { Modulus }\left(10^{6} \mathrm{~N} / \mathrm{m}^{2}\right)\end{array}$ & $\begin{array}{c}\text { Poisson's } \\
\text { ratio }\end{array}$ \\
\hline Epoxy & 1180 & 4350 & 0.3679 \\
Steel & 7800 & 210000 & 0.29 \\
Rubber & 1300 & 0.1175 & 0.47 \\
\hline \hline
\end{tabular}

Through use of the finite element method ${ }^{[24]}$, the systems described in Fig. 1 were studied theoretically. The band structures and displacement vector fields were computed according to the Bloch theorem. The single unit cell is determined by the periodicity of the structure. The following geometric parameters were used: $D=8 \mathrm{~mm} ; e=1 \mathrm{~mm} ; a=10 \mathrm{~mm} ; h=5 \mathrm{~mm}, h_{R}=2.5 \mathrm{~mm}$, $h_{S}=2.5 \mathrm{~mm}$, and $d=7 \mathrm{~mm}$. The material parameters used in the calculation are shown in Table 1.
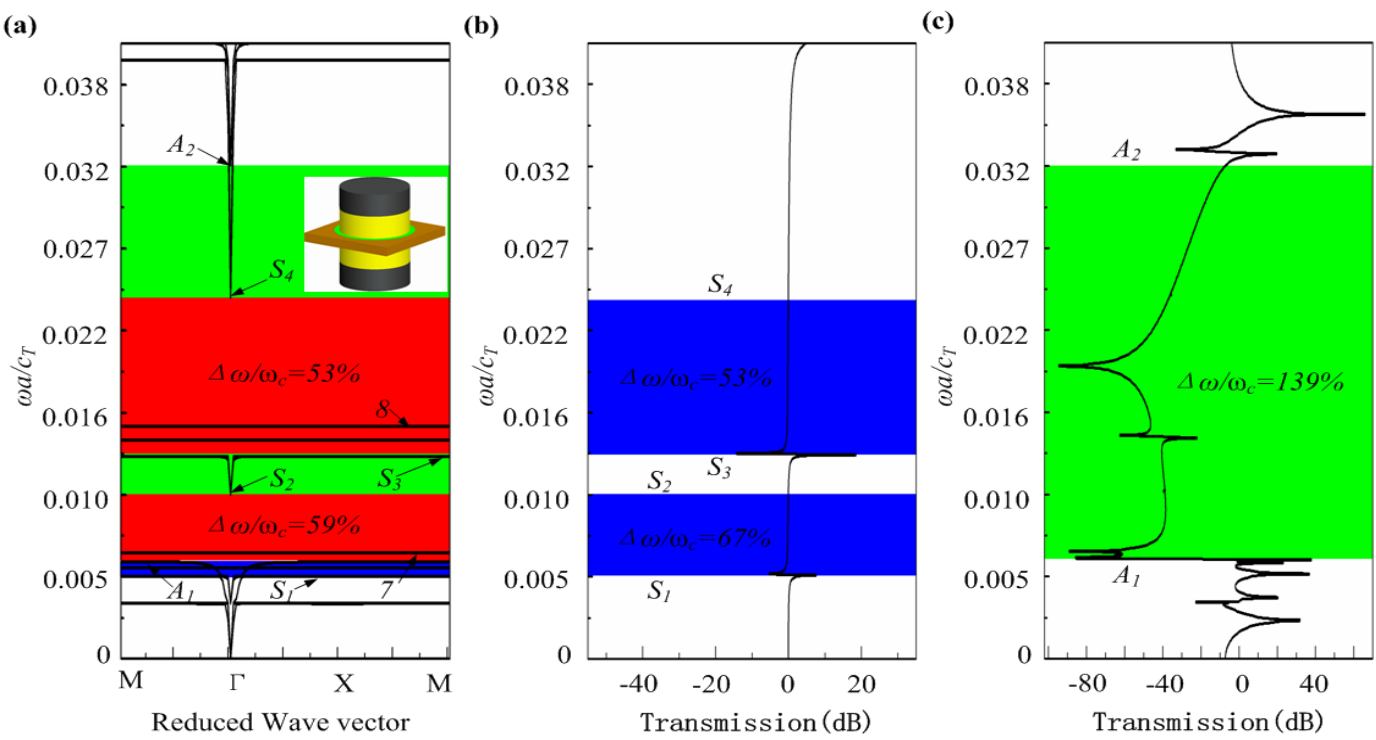

Fig. 2. Schematics of the band structure and transmission power spectra ( $6 \times 1$ unit cells) of the proposed structure: (a) band structure; (b) transmission power spectra of the in-plane waves; and (c) transmission power spectra of the out-of-plane waves. The inset is an illustration of the unit cell. The red, blue, and green shaded regions denote the complete, in-plane, and out-of-plane band gaps, respectively.

The band structure of the proposed structure is illustrated in Fig. 2(a). Fifteen bands are contained in the normalized frequency range of $0-0.04$, and can be classified into the traditional plate modes ${ }^{[32]}$ or the flat modes. The traditional plate modes include the in-plane modes (mainly the symmetric Lamb modes, e.g. $S_{2}$ and $S_{4}$ ) and the out-of-plane modes (mainly the anti-symmetric Lamb modes, e.g. $A_{2}$ ). Moreover, the flat modes (e.g. $S_{1}, S_{3}, A_{1}, 7$, and 8 ) are the resonant modes of the composite stubs. The band gaps are a result of the coupling between the two kinds of modes mentioned above. To determine the number of band gaps, we first investigated the influence of the special flat bands on the band gaps. The mode displayed in Fig. 3 corresponds to the 7th flat band. The corresponding vibration is mainly due to stub rotation and non-coupling with the plate. This means that no reacting force was applied to the plate. Therefore, these special types of flat bands have no relationships to the band gaps. 


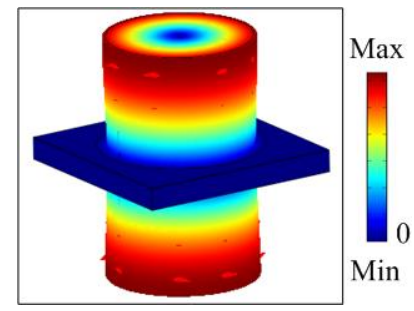

Fig. 3. The total displacement vector fields of the mode corresponding to the 7th flat band of the proposed structure.

The first out-of-plane band gap (green shaded regions shown in Fig. 2) is caused by the coupling between the out-of-plane mode (mode $A_{2}$ ) and the corresponding flat mode (mode $A_{1}$ ), and ranges from 0.006 to 0.033 (between the 6th and 15th bands). The relative band width, $\Delta \omega / \omega$, is defined as the ratio between the band gap width and the center frequency of the band. The relative bandwidth of the out-of-plane band gap is equal to $139 \%$. There are two in-plane band gaps (blue shaded regions shown in Fig. 2), and they are caused by the coupling between the in-plane modes (modes $S_{2}$ and $S_{4}$ ) and the corresponding flat modes (modes $S_{1}$ and $S_{3}$ ). The first band gap ranges from 0.0054 to 0.011 (between the 4th and the 8 th bands) and the second band gap ranges from 0.014 to 0.024 (between the 9 th and the 14th bands). The relative bandwidth of the first and second in-plane band gaps are equal to $67 \%$ and $53 \%$, respectively. Furthermore, there are two complete band gaps (red shaded regions shown in Fig. 2), and they are caused by overlap between both the first and the second in-plane band gaps and the first out-of-plane band gap. The first band gap ranges from 0.006 to 0.011 (between the 6th and the 8th bands), and the second band gap ranges from 0.014 to 0.024 (between the 9th and the 14th bands). The relative bandwidths of the first and second complete band gaps are equal to $59 \%$ and $53 \%$, respectively.

(a)

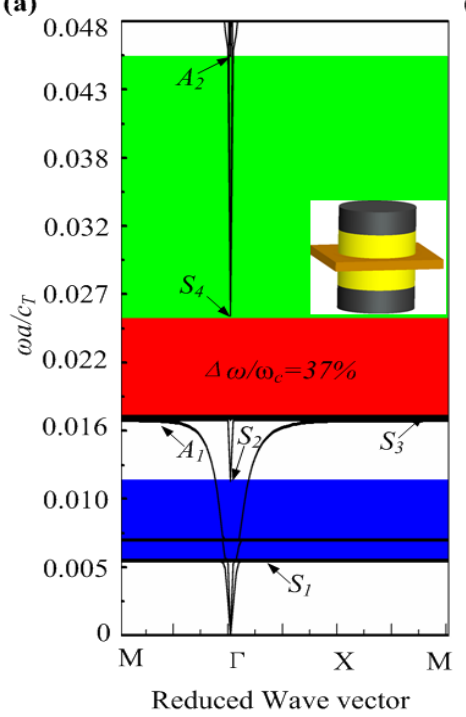

(b)

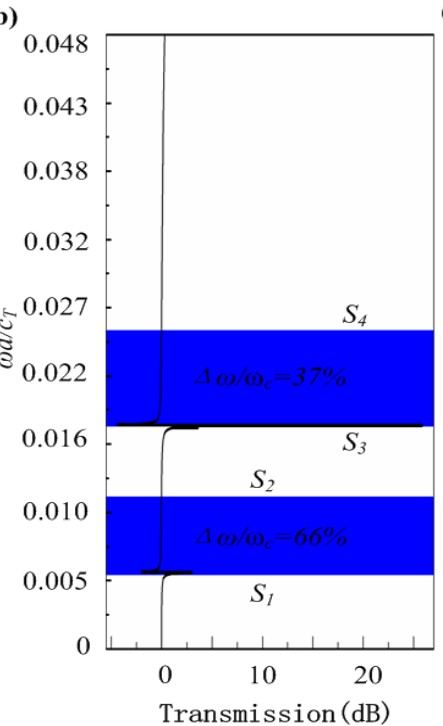

(c)

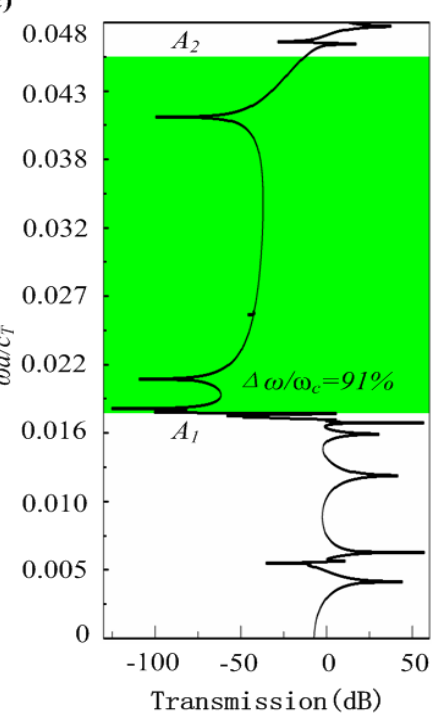

Fig. 4. Schematics of the band structure and transmission power spectra ( $6 \times 1$ unit cells) of the classic structure: (a) band structure; (b) transmission power spectra of the in-plane waves; and (c) transmission power spectra of the out-of-plane waves. The inset is an illustration of the unit cell. The red, blue, and green shaded regions denote the complete, in-plane, and out-of-plane band gaps, respectively.

As a comparison, we also calculated the band structure of the classic structure sans rubber filler, as shown in Fig. 4(a). There are two in-plane band gaps (the blue shaded regions shown in Fig. 4) and one out-of-plane band gap (the green shaded regions shown in Fig. 4). The relative 
bandwidths of the first and the second in-plane band gaps are equal to $66 \%$ and $37 \%$, respectively. The relative bandwidth of the out-of-plane band gap is equal to $91 \%$. The complete band gap (red shaded regions shown in Fig. 4) is due to the overlap between the first out-of-plane band gap and the second in-plane band gap. The relative bandwidth is equal to $37 \%$.

TABLE 2.Results comparison.

\begin{tabular}{llllll}
\hline \hline & \multicolumn{4}{c}{ Proposed structure } & Classic structure \\
\cline { 2 - 5 } Relative & In-plane band & 1th & $67 \%$ & $\Longleftrightarrow$ & $66 \%$ \\
bandwidth & Out-of-plane & 2nd & $53 \%$ & & $37 \%$ \\
& band gaps & 1th & $139 \%$ & $\Uparrow$ & $91 \%$ \\
& Complete band & 1th & $59 \%$ & $\Uparrow$ & $37 \%$ \\
& gaps & 2nd & $53 \%$ & $\Uparrow$ & 0 \\
\hline \hline
\end{tabular}

From Table 2, it is clear that the introduction of the rubber filler into the proposed structure results in little change to the in-plane band gaps, but results in a large change to the out-of-plane band gap; particularly in comparison to the classic structure. The expanded out-of-plane gap overlaps the first in-plane band gap, thus generating a new complete band gap. As a result, the complete band gaps in the lower frequency ranges are expanded. The expanded complete band gap is mainly due to the expansion of the out-of-plane band gap. Moreover, the expansion of the out-of-plane band gap is caused by the rubber filler, which is capable of shifting the out-of-plane band gap into lower frequency ranges and maintaining the bandwidth.

\section{A. Formation mechanism of the lower-frequency out-of-plane band gap}

In order to study the physical mechanism for the expanded lower-frequency complete band gap in the proposed structure, several modes were extracted: resonance modes $A_{l}$ and $S_{l}$, which correspond to the lower edge of the first band gap (out-of-plane and in-plane band gap); and traditional plate modes $A_{2}$ and $S_{2}$, which correspond to the upper edge of the first band gap (in-plane and out-of-plane band gaps).
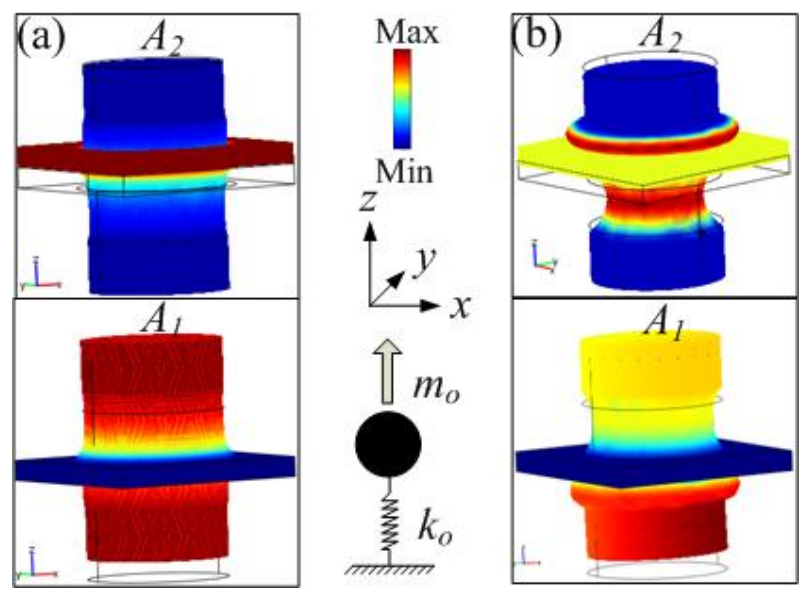

Fig. 5. The total displacement vector fields of the modes (resonant mode $A_{l}$ and anti-symmetric Lamb mode $A_{2}$ ):

(a) corresponding to Fig. 2(a); and (b) corresponding to Fig. 4(a).

The total displacement vector in a unit cell of the proposed structure and the classic structure 
are shown in Fig. 5(a) and Fig. 5(b), respectively. These modes correspond to the upper and lower edge of the out-of-plane band gap in each structure. The mode $A_{2}$ is an anti-symmetric Lamb mode of the plate. The epoxy plate vibrates along the $z$-axis while the stub remains stationary. At certain frequencies, the anti-symmetric Lamb mode will be activated and the out-of-plane waves will propagate through the PC plate in the anti-symmetric Lamb mode. When the frequency of the out-of-plane waves is near the first natural frequency of the stub resonator, the resonant mode, $A_{I}$ (flat mode), will be activated. The stub vibrates along the $z$-direction and applies a reacting force to the plate. In this scenario, the out-of-plane waves are not capable of propagating through the PC plate, and an out-of-plane band gap is formed.

The opening location of the out-of-plane band gap is determined by the natural frequency of the resonant mode, $A_{l}$. As shown in Fig. 5 , the vibration process of the resonant mode, $A_{l}$, can be understood as a mass-spring system (" $k_{o}-m_{o}$ "), where $k_{o}$ is the tension and compression stiffness of the spring and $m_{o}$ is the mass. For the classic structure, the rubber stub acts as the spring and the steel stub acts as the mass. For the proposed structure, the displacement fields are distributed throughout and manifest as an "analogous-rigid mode" of the whole stub, since the whole stub bodily moves along the z-axis with weak constraint. This "analogous-rigid mode" of the stub can be considered a mode generated by strengthening the local resonance effect of the composite plate through the introduction of double stubs. In this case, the rubber filler acts as a spring and the whole stub acts as a mass. Because the stiffness of the rubber stub is weaker than that of the rubber filler and the mass of the whole stub is larger than that of one steel stub, the opening location of the out-of-plane band gap is shifted into a lower frequency range and overlaps with the first in-plane band gap. Therefore, the relative band width of the out-of-plane band gap is expanded.

As shown in Fig. 6(a), we verified the above analysis using the eigenmode of the out-of-plane band gap when the frequency of the out-of-plane waves were 0.006. The plate was nearly stationary, and the whole stub vibrates in an "analogous-rigid mode" along the $z$-direction. It leads the out-of-plane wave attenuation in the band gaps.

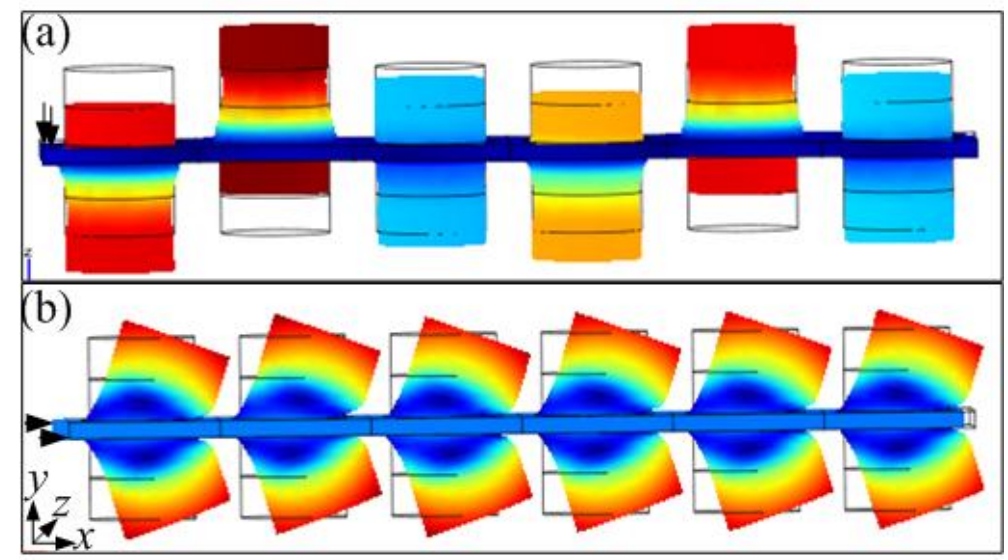

Fig. 6. Schematics of the eigenmodes: (a) the out-of-plane band gap when the frequency of the out-of-plane waves was 0.006; and (b) the in-plane band gap when the frequency of the in-plane waves was 0.006 .

Fig. 7 displays the total displacement vectors magnitudes in a unit cell of the proposed structure and the classic structure. These magnitudes correspond to the upper and lower edges of the first in-plane band gap of each structure. The in-plane band gap is formed due to coupling between the resonant mode, $S_{1}$, of the stub and the symmetric Lamb mode, $S_{2}$, of the plate. The 
corresponding vibration of the resonant mode, $S_{1}$, of the stub is mainly from the bending of the stubs. The opening location is determined by the natural frequency of the resonant mode, $S_{1}$. The resonant mode, $S_{l}$, can also be understood as a mass-spring system (" $\left.k_{i}-m_{i}{ }^{\prime \prime}\right)$, as shown in Fig. 6. In this mass-spring system, $k_{i}$ is the torsion stiffness of the spring and $m_{i}$ is the mass. For the two PC plates, the rubber stub acts as a spring and the steel stub acts as a mass, so the openings of the in-plane band gap are located in approximately the same frequency range. Therefore, it can be concluded that the expansion of the band gap opening in lower frequencies is due to the stronger coupling between the "analogous-rigid mode" of the stubs and the anti-symmetric Lamb modes of the plate.
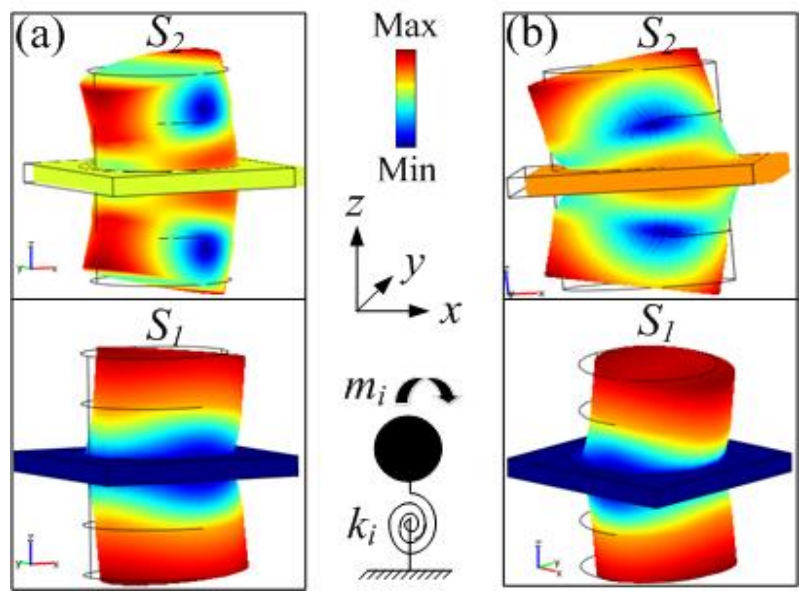

Fig. 7. The total displacement vector fields of the modes (resonant mode $S_{1}$, symmetric Lamb mode $S_{2}$ ): (a) corresponds to Fig. 2(a) and (b) corresponds to Fig. 4(a).

As shown in Fig. 6, we verified the above analysis using the eigenmode of the in-plane band gap when frequency of the in-plane waves was 0.006 . The plate was nearly stationary and stubs ware bending, which lead to in-plane wave attenuation in the band gaps.

We can conclude from the above investigations that the resonator in the PC plate can be considered as a spring-mass system, as shown in Fig. 8. This spring-mass system includes two subsystems. The first one is the " $k_{o}-m_{o}$ " subsystem, as shown in Fig. 5. Its local resonance mode is coupled with the anti-symmetric Lamb mode of the plate and formed out-of-plane band gaps. The second one is the " $k_{i}-m_{i}$ " subsystem, as shown in Fig. 7. Its local resonance mode is coupled with the symmetric Lamb mode of the plate, and formed the in-plane band gaps. The two types of band gaps overlap each other to form the complete band gaps. The opening locations of the in-plane and out-of-plane band gaps depend on the natural frequencies of the two subsystems.

(a)

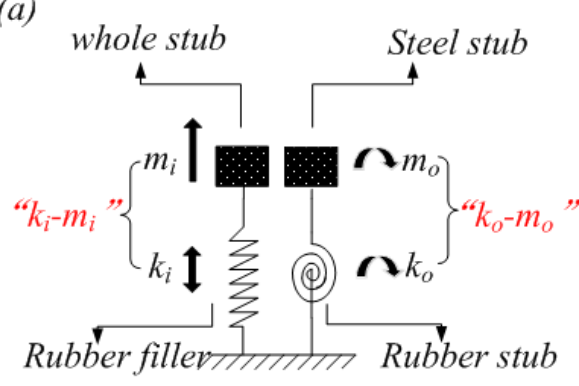

(b)

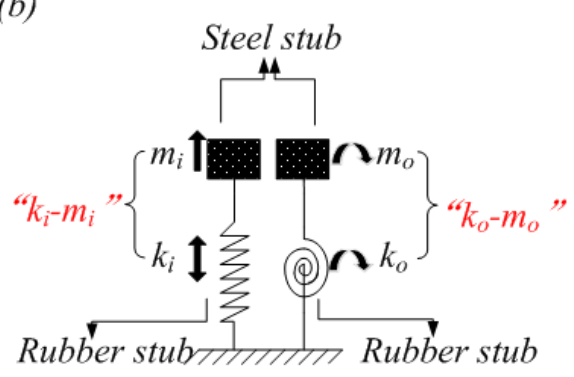

Fig. 8. Spring-mass equivalent systems of (a) the proposed structure and (b) the classic structure.

Considering the classic PC plate, the steel stub acts as a mass and the rubber stub acts as a spring, which leads to a coupling between the " $k_{o}-m_{o}$ " subsystem and the " $k_{i}-m_{i}$ " subsystem. 
Therefore, it is difficult to adjust the in-plane and out-of-plane band gaps independently. In the proposed structure, the rubber filler acts has a stiffness, $k_{o}$, the rubber stub has a stiffness, $k_{i}$, the whole stub acts as the mass, $m_{o}$, and the steel stub acts as the mass, $m_{i}$. As a result, the " $k_{o}-m_{o}$ " subsystem and the " $k_{i}-m_{i}$ " subsystem can be decoupled. Moreover, the mass, $m_{o}$, is magnified due to the "analogous-rigid mode" of the whole stub. Therefore, the out-of-plane band gap can be adjusted into the lowest frequency range. As a result, the two band gaps can be overlapped with each other in the lowest frequency, forming a lowest-complete band gap with an expanded relative band width.

\section{B. The effect of the composite stubs on the first band gap}

In order to investigate the effect of the composite stubs on the complete BGs of the proposed structure, we studied the influence of the steel stub height on the first complete BG. Fig. 9 displays the evolution of the first complete $\mathrm{BG}$ as a function of the steel stub height $h_{S}$.

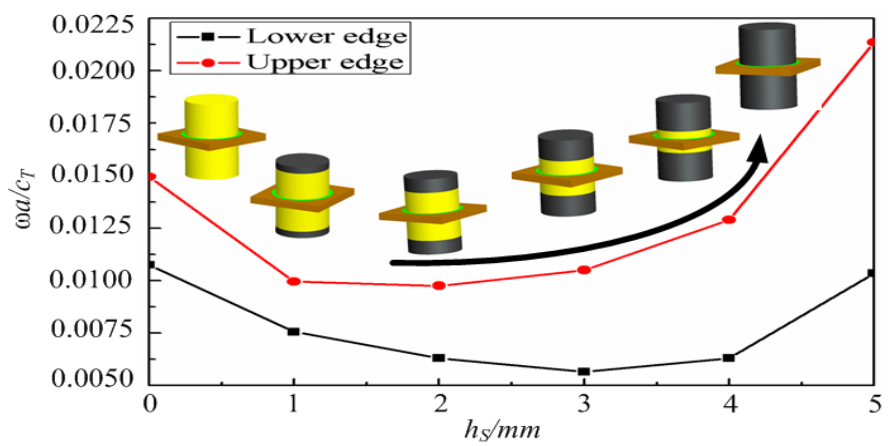

Fig. 9. The evolution of the first complete BG in the proposed structure as a function of the steel stub height with $D=8 \mathrm{~mm}, d=7 \mathrm{~mm}, h=5 \mathrm{~mm}, e=1 \mathrm{~mm}$, and $a=10 \mathrm{~mm}$, respectively. The insets are the schematic view of the unit cell of the considered systems with different stub heights.

We can find that with the increase of the steel stub height both the lower and upper edges of the first complete band gap shift to a lower frequency range and move to higher frequency range. For example, when the steel stub height is less than or equal to $3 \mathrm{~mm}$, the lower edge shifts to lower frequencies as the steel stub height increases. However, when the steel stub height is larger than $3 \mathrm{~mm}$, the lower edge frequency shifts to higher frequencies as the steel stub height increases. This phenomenon is due to the "analogous-rigid mode" of the stubs, and changes with the steel stub height. Consider the displacement field of the mode corresponding to the lower edge of the first complete band gap with different steel stub heights. As shown in Fig. 10, when the steel stub height is less than or equal to $3 \mathrm{~mm}$, the displacement field is distributed throughout the stub and manifests the "analogous-rigid mode". The "analogous-rigid mode" of the stub vibrates along the $z$-axis direction and exhibits a stronger coupling with the anti-symmetric Lamb mode of the plate. In this case, the complete band gap opening is determined by the out-of-plane band gap. As the height of the steel stub increases, the lumped mass increases while the spring stiffness remains constant; this causes the lower edge of the first complete band gap to shift to lower frequencies. When the steel stub height is larger than $3 \mathrm{~mm}$, the "analogous-rigid mode" of the stubs vibrates along the $x y$-plane and couples with the symmetric Lamb mode of the plate. In this case, the complete band gap opening is determined by the in-plane band gap. As steel stub height increases, both the lumped mass and the spring stiffness increase. However, the spring stiffness increases more, which causes the lower edge of the first complete band gap to shift to higher frequencies. 


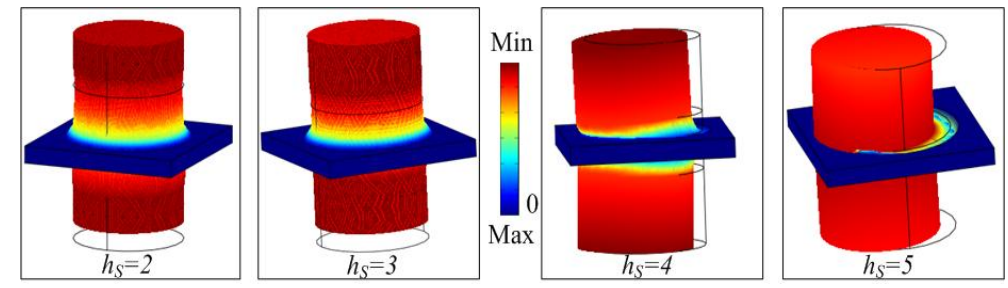

Fig. 10. The displacement fields of the modes of the lower edge of the first band gap of the proposed structure with different steel stub heights.

\section{Conclusions}

In this paper, we presented an original structure that significantly expanded the relative bandwidth of the complete band gap. The proposed structure is a type of two-dimensional locally resonant PCs and consists of a double-sided stubbed composite plate with composite stubs. Compared to the classic double-sided stubbed PC plate with composite stubs, we showed that the relative bandwidth was expanded by a factor of 1.5 , and that the opening position of the first complete band gap was decreased by a factor of 3 . In addition, we showed that more band gaps can be obtained. These phenomena are due to the "analogous rigid mode" of the stub. The mechanism can be summarized as follows:

1) The "analogous-rigid mode" of the stub is generated due to the enhancement of the locally resonant effect of the composite plate after introducing double stubs. Moreover, the analogous-rigid mode can be strengthened by introducing composite stubs.

2) The expansion of the band gap opening in lower frequencies is due to the stronger coupling between the "analogous-rigid mode" of the stubs and the anti-symmetric Lamb modes of the plate. The spring-mass system of the resonator was decoupled by introducing rubber filler, allowing the out-of-plane band gaps to be adjusted separately. The in-plane band gaps remained relatively unchanged while the out-of-plane band gapswere greatly expanded. The expanded out-of-plane gap overlapped the first in-plane band gap, and thus generated a new complete band gap. As a result, the complete band gaps in the lower frequency ranges were expanded.

Finally, we investigated the effects of the composite stubs on the band gaps. Results show that the location of the complete band gaps can be modulated in a significant lower frequency range and the bandwidth can be expanded in a considerable large frequency range by introducing different composite stubs. Those results show that it is possible to build simple LR structures with a relatively large local resonant band gap in lower frequencies for sound insulation and vibration control applications.

\section{Acknowledgements}

This research was supported by the Project of National Natural Science Foundation of China (No.51275377) and the Program for Changjiang Scholars and Innovative Research Team in University (No.IRT1172). We would like to thank Dr. Linkai Niu for his kind comments to improve the paper.

\section{References}

[1] M. S. Kushwaha, P. Halevi, L. Dobrzynski, and B. Djafari-Rouhani, Phys. Rev. Lett. 71(13), 2022-2025 (1993).

[2] R. Martínez-Sala, J. Sancho, J. V. Sánchez, V. Gómez, J. Llinares, and F. Meseguer, 
Nature(London) 378, 241(1995).

[3] J.O. Vasseur, P. A. Deymier, B. Chenni, B. Djafari-Rouhani, L. Dobrzynski, D. Pre-vost, Phys. Rev. Lett. 86 (2001) 3012.

[4] S. Benchabane, A. Khelif, J.-Y. Rauch, L. Robert, and V. Laude, Phys. Rev. E 73,065601(R)(2006).

[5] S. Mohammadi, A. A. Eftekhar, W. D. Hunt, and A. Adibi, Appl. Phys. Lett. 94, 051906 (2009).

[6]T.-T. Wu, J.-C. Hsu, and J.-H. Sun, IEEE Trans. Ultrason. Ferroelectr. Freq. Control 58, 2146 (2011).

[7] J.H. Ma, Z.L. Hou, B. M. Assouar, J. Appl. Phys. 115 (2014) 093508.

[8] M. S. Kushwaha, P. Halevi, L. Dobrzynski, B. Djafari-Rouhani, Phys. Rev. Lett. 71 (1993) 2022.

[9] T. Gorishnyy, C. K. Ullal, M. Maldovan, G. Fytas, E. L. Thomas, Phys. Rev. Lett. 94 (2005) 115501.

[10] T. C. Wu, T. T. Wu, J. C. Hsu, Phys. Rev. B 79 (2009)104306.

[11] X.F. Zhu, Phys. Lett. A 377 (2013) 1784.

[12] Z. Liu, X. Zhang, Y. Mao, Y.Y. Zhu, Z. Yang, C.T. Chan, P. Sheng, Science 289 (2000) 1734.

[13] C. Goffaux, J.Sánchez-Dehesa, A. L. Yeyati, P. Lambin, A. Khelif, J. O. Vasseur, B. Djafari-Rouhani, Phys. Rev. Lett. 88 (2002) 225502.

[14] O. Sigmund and J. S. Jensen, Philos. Trans. R. Soc. A 361(1806), 1001-1019 (2003).

[15] M. Hirsekorn, Appl. Phys. Lett. 84 (2004) 3364.

[16] M. I. Hussein, K. Hamza, G. M. Hulbert, R. A. Scott, and K. Saitou, Struct. Multidiscip. Optim. 31, 60-75 (2006).

[17] Y. Pennec, B. Djafari-Rouhani, H. Larabi, J.O. Vasseur, A.C. Hladky-Hennion, Phys. Rev. B 78 (2008) 104105.

[18] T.T. Wu, Z.G. Huang, T.C. Tsai, T.C. Wu, Appl. Phys. Lett. 93 (2008) 111902.

[19] X. Zhou, G. Hu, Phys. Rev. B 79 (2009) 195109.

[20] Y. Pennec, B. Djafari-Rouhani, H. Larabi, J. O. Vasseur, and A. C. Hladky-Hennion, Phys. Rev. B 78(10), 104105 (2008).

[21] C.Y. Sun, J.C. Hsu, T.T. Wu, Appl. Phys. Lett. 97 (2010) 031902.

[22] O. R. Bilal and M. I. Hussein, Phys. Rev. E 84, 065701 (2011).

[23] M. B. Assouar, M. Senesi, M. Oudich, M. Ruzzene, Z. Hou, Appl. Phys. Lett. 101(17) (2012) 173505.

[24] J. H. Wen, D. L. Yu, G. Wang, H. G. Zhao, Y. Z. Liu, and X. S. Wen, Chin. Phys. Lett. 24, 1305 (2007).

[25] A. Khelif, B. Aoubiza, S. Mohammadi, A. Adibi, and V. Laude, Phys. Rev. E 74, 046610 (2006).

[26] J. J. Chen, X. Han, and G. Y. Li, J. Appl. Phys. 113, 184506 (2013).

[27] J. Chen, Y. Xia, X. Han, and H. Zhang, Ultrasonics 52, 920 (2012).

[28] J. Zhu, Y. Chen, X. Zhu, J. Garcia-Vidal, X. Yin, W. Zhang, X. Zhang, Sci. Rep. (2013) 3.

[29]J.C. Hsu, T.T. Wu, Appl. Phys. Lett. 90 (2007) 201904.

[30] T.T. Wu, Z.G Huang, T. C. Tsai. T. C. Wu, Appl. Phys. Lett. 93 (2008) 111902.

[31]O.R. Bilal, M.I. Hussein, Appl. Phys. Lett. 103 (2013) 111901. 
[32] Y. G. Li, T. N. Chen, X. P. Wang, Y. H. Xi, Q. X. Liang, Phys. Lett. A379 (2015) 412416.

[33] B.M. Assouar, M. Oudich, Appl. Phys. Lett. 100 (2012) 123506.

[34] H. J. Zhao, H. W. Guo, B. Y. Li, Z. Q. Deng, R. Q. Liu, J. Appl. Phys. 118, 044906 (2015). 